\title{
Tunable Amplification and Cooling of a Diamond Resonator with a Microscope
}

\author{
Harishankar Jayakumar, ${ }^{* \dagger, \dagger}$ Behzad Khanaliloo, ${ }^{\dagger, \S}$ David P. Lake $\odot,{ }^{\natural}$ and Paul E. Barclay" \\ Institute for Quantum Science and Technology, University of Calgary, Calgary, Alberta T2N 1N4, Canada
}

(Received 20 February 2021; accepted 10 March 2021; published 27 July 2021)

\begin{abstract}
Control of the dynamics of mechanical resonators is central to quantum science and metrology applications. Optomechanical control of diamond resonators is attractive owing to the excellent physical properties of diamond and its ability to host electronic spins that can be coherently coupled to mechanical motion. Using a confocal microscope, we demonstrate tunable amplification and damping of the motion of a diamond nanomechanical resonator. Observation of both normal-mode cooling from room temperature to $80 \mathrm{~K}$ and amplification into self-oscillations with $60 \mu \mathrm{W}$ of optical power is observed via waveguide optomechanical readout. This system is promising for quantum spin optomechanics, as it is predicted to enable optical control of stress-spin coupling with rates of approximately $1 \mathrm{MHz}(100 \mathrm{THz})$ to ground (excited) states of diamond nitrogen-vacancy centers.
\end{abstract}

DOI: 10.1103/PhysRevApplied.16.014063

\section{INTRODUCTION}

The interaction between light and mechanical systems underlies breakthroughs in physics ranging from optical tweezers [1] to gravitational-wave detection [2]. Nanoscale systems harnessing this interaction have led to advances in quantum nanomechanics [3-9], sensing [10-13], and nonlinear optics [14-18]. An essential ingredient of many of these demonstrations is dynamic optomechanical back action, which allows energy exchange between the optical and mechanical domains $[19,20]$. The control of diamond nanomechanical systems via optomechanical back action is of growing interest, fueled by the exceptional properties of diamond [21] and by demonstrations of diamond spin manipulation using piezoelectronically driven mechanical resonators [22-24,24-31]. Actuating resonator motion optomechanically provides a path toward photon-phononspin coupling and technologies ranging from spin-spin entanglement [32,33] to quantum transduction [34]. Back action can also enhance the performance of diamond resonators used for sensing [35-37]. In this paper, we show that optomechanical back action acting on modes of a diamond resonator vibrating in or out of plane can be selectively created and controlled by adjusting the focal position of a microscope that commonly serves as an

\footnotetext{
*harish@umn.edu

${ }^{\dagger}$ These authors contributed equally.

$¥$ Current address: University of Minnesota, 321 Church Street SE, Minneapolis, Minnesota 55455, USA.

${ }^{\S}$ Current address: Rockley Photonics Inc.

"Current address: California Institute of Technology, $1200 \mathrm{E}$ California Boulevard, Pasadena, California 91125, USA.

"pbarclay@ucalgary.ca
}

optical interface with diamond color centers. Using this technique, we cool a diamond nanomechanical resonator, as well as amplify its motion sufficiently for mechanical control of diamond spins via their predicted coupling to phonons.

Optomechanical damping and amplification - for example, by optical gradient [38], radiation pressure [4,39,40], or photothermal [36,41-45] forces - typically relies on feedback from a cavity $[19,20]$, waveguide coupler [45], or external optoelectronics [46]. Inspired in part by optical tweezers, here we introduce a system that operates in a regime of optomechanics dominated by optical intensity gradient backaction and does not require a cavity or coupling to optical resonances [44]. The dynamic optomechanical back action is photothermal in nature and is tuned through translation of a microscope focus, allowing both the strength and the sign of the optomechanical damping to be adjusted. This system, which adds confocal microscopy to our previously demonstrated waveguide optomechanical experiment [45], provides a combination of tunable optomechanical actuation and sensitive optomechanical readout. It allows normal-mode cooling of a diamond mechanical resonator from room temperature to approximately $80 \mathrm{~K}$ and excitation of nanomechanical self-oscillations, the stress field of which is predicted to allow control of nitrogen-vacancy- $(\mathrm{N}-V)$ center spins [22]. These self-oscillations are observed for continuouswave excitation from a $532-\mathrm{nm}$ laser with a power as low as $60 \mu \mathrm{W}$. Unlike previous demonstrations of photothermal back action [36,41-44], neither an external cavity nor a wavelength-tunable laser is required to adjust the back action between the damping and antidamping regimes. Furthermore, we leverage its sensitivity to the microscope field gradient to selectively excite vertical as 
well as horizontal modes of the nanobeam, the latter of which can be difficult to probe using conventional optical interferometry measurements of nanomechanical devices [47], as their motion primarily induces intensity rather than phase changes on reflected light.

\section{DEVICE AND EXPERIMENTAL SETUP}

The optomechanical system studied here, illustrated in Fig. 1(a), consists of a diamond nanobeam (dimensions $\left.l \times w \times t=50 \times 0.5 \times 0.25 \mu \mathrm{m}^{3}\right)$ illuminated by a green (532-nm) laser input to an objective (Sumitomo long working distance, $0.55 \mathrm{NA}$ ) mounted on a three-axis stage. The nanobeam is fabricated from single-crystal diamond (Element Six, optical grade, $3 \times 3 \mathrm{~mm}^{2}$ area, polished by Delaware Diamond Knives) using undercut etching [45] and its top surface is coated with titanium (approximately 5-nm thick, deposited using electron-beam evaporation), which enhances the photothermal effects discussed below. The nanobeam is suspended approximately $2 \mu \mathrm{m}$ above the diamond substrate, as shown in Fig. 1(a).

In the results presented below, we show that translating the microscope controls the dynamics of the motion of the nanobeam. These dynamics are monitored using an optical fiber-taper waveguide (diameter approximately
$1 \mu \mathrm{m})$ [48] evanescently coupled to the nanobeam, as illustrated in Fig. 1(a). The fiber taper and the diamond sample are mounted in a closed-cycle cryostat (Montana Instruments) operating in high vacuum over temperatures from $5 \mathrm{~K}$ to $300 \mathrm{~K}$ and are aligned using nanopositioners (Attocube). Nanobeam motion is monitored with up to $\mathrm{fm} / \sqrt{\mathrm{Hz}}$ sensitivity by detecting fluctuations in the coupling between the fiber taper and the nanobeam, as described in Ref. [45]. The nanobeam resonance dynamics are measured from the power spectral density $S_{v v}$ of the photodetected transmission of a $1570-\mathrm{nm}$ source through the fiber taper.

The characterization of the fundamental nanobeam vertical mechanical resonance $\left(\mathbf{v}_{1}\right)$ in the absence of the microscope field is shown in Fig. 1(b), which plots $S_{v v}$ over the frequency $(f)$ range spanning $\mathbf{v}_{1}$ resonance frequency $f_{m}$, in high vacuum $\left(<10^{-5}\right.$ Torr $)$ at $300 \mathrm{~K}$ and $5 \mathrm{~K}$ operating temperatures. The peak in $S_{v v}$ is thermally driven motion of $\mathbf{v}_{1}$, the dynamics of which are determined by dissipation rate $\Gamma_{m}=2 \pi f_{m} / Q_{m}$, where $Q_{m}$ is mechanical quality factor. Fitting $S_{v v}$ with a thermomechanical noise spectrum [49], we find $Q_{m}=7.5 \times 10^{4}$ and $5.8 \times 10^{5}$ at $300 \mathrm{~K}$ and $5 \mathrm{~K}$, respectively. The fiber-taper input power is sufficiently low (a few microwatts) so that it does not affect the dynamics.
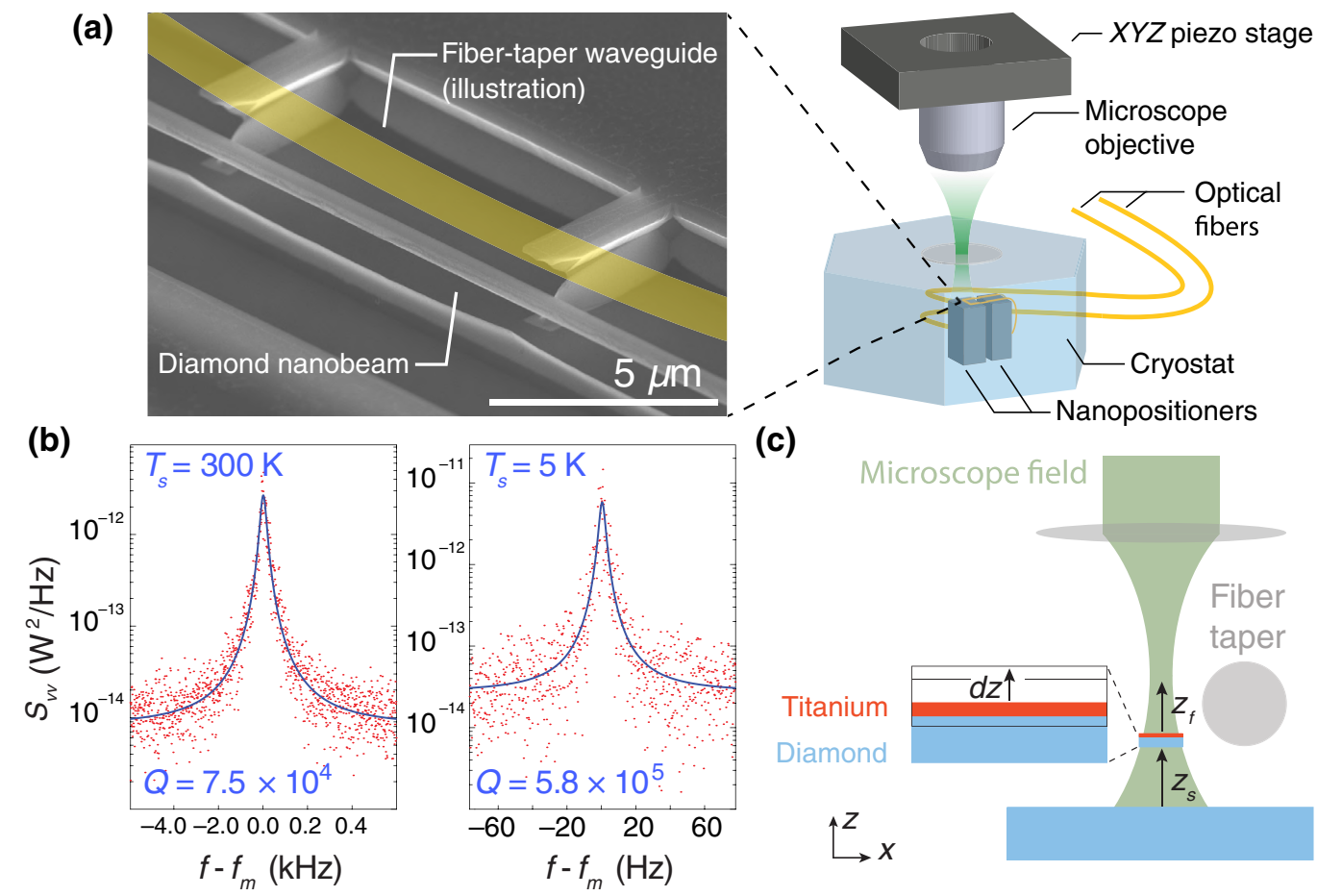

(c)

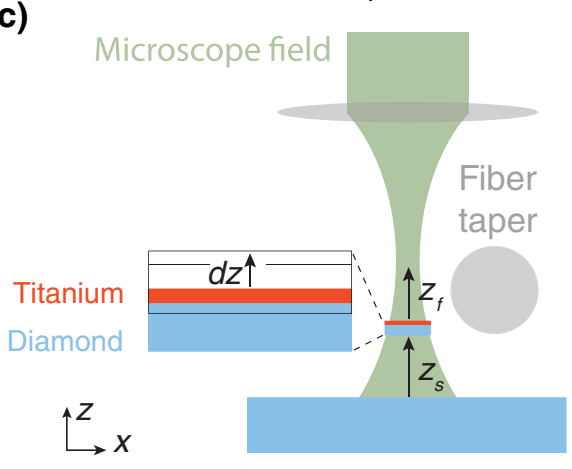

FIG. 1. (a) A schematic of the optomechanical system and apparatus. A microscope objective mounted on a piezo stage focuses a green laser onto the sample. The sample and fiber taper are located in a cryostat on nanopositioners. The SEM image shows a diamond nanobeam similar to that used in the experiment, with an illustration of the dimpled optical fiber taper drawn in yellow, in approximately the position used for evanescent coupling to the nanobeam. Also visible are the diamond supports used to stabilize the fiber taper during the measurements. (b) The power spectral density of the fiber-taper transmission near the $\mathbf{v}_{1}$ nanobeam mode frequency at room and low temperature. (c) A schematic of the geometry of the optomechanical system. 


\section{TUNABLE OPTOMECHANICAL BACK ACTION}

The action of turning on the microscope field introduces optomechanical back action that can be analyzed using the geometry in Fig. 1(c). The field intensity $I$ in the nanobeam depends on both the height of the nanobeam above the substrate, $z_{s}$, and its distance to the microscope focal plane, $z_{f}$, and can be approximated as $I=I_{f}\left(z_{f}\right) \chi\left(z_{s}\right)$. Here, $I_{f}$ describes the $z_{f}$ dependence of $I$. The etalon enhancement factor $\chi\left(z_{s}\right)$ describes interference between reflections from the etched diamond surface below the nanobeam, the titanium-coated nanobeam, and the incident field, which will combine to create a standing wave pattern. In this simplified model, $I_{f}\left(z_{f}\right)$ implicitly accounts for geometry-related local field corrections - for example, local optical resonances of the nanobeam and the effect of the titanium layer - and we assume that changes in $z_{s}$ from nanobeam motion are sufficiently small for the etalon contribution to be treated as a separable scaling factor. The vertical nanobeam displacement $d z$ modifies $z_{s}$ and $z_{f}$ by $\pm d z$, respectively, which in turn changes $I$. This optomechanical feedback, when combined with a lag between the nanobeam position and forces proportional to $I$, amplifies or damps the mechanical motion.

The dominant optical microscope forces on the nanobeam are found to be photothermal $[36,42]$, and their optomechanical damping $\Delta \Gamma_{m}$ is given by

$$
\frac{\Delta \Gamma_{m}\left(z_{f}\right)}{\Gamma_{m}^{o}}=Q_{m}^{o} \frac{2 \pi f_{m}^{o} \tau}{1+\left(2 \pi f_{m}\left(z_{f}\right) \tau\right)^{2}} \mathcal{G} \frac{d I}{d z} \sigma
$$

where $f_{m}^{o}, \Gamma_{m}^{o}$ and $Q_{m}^{o}$ are intrinsic values in the absence of the microscope field. This model follows and modifies that previously analyzed in Ref. [45] in the absence of a microscope field. Unlike in Ref. [45], the field from the fiber taper does not heat the nanobeam sufficiently to induce any back action. Instead, the nanobeam is deflected by power absorbed from the microscope field. The nanobeam deflection for absorbed power $\sigma I$ is determined by the photothermal coupling coefficient $\mathcal{G}$ (in units of $\mathrm{m} / \mathrm{W}$ ) and depends on the geometry and internal compressive stress of the nanobeam [45]. The titanium layer increases the absorption cross section $\sigma$ but is not generally necessary to observe dynamic back action [45]. A noninstantaneous thermal response time $\tau$ is required for optomechanical heating or cooling. Finite-element (COMSOL) simulations predict $2 \pi f_{m} \tau \sim 3$, accounting for the reduced thermal conductivity of nanostructured diamond [50].

The gradient of the microscope intensity plays a critical role in determining whether $d I / d z$, and as a result $\Delta \Gamma_{m}$, is positive or negative. This is in contrast to cavity optomechanics, where back action is dominated by $\chi$, the sign of which is independent of the external optics. To study the microscope back action, the objective is aligned with the center of the nanobeam and scanned vertically ( $1 \mu \mathrm{m}$ steps, 2.9 s per step) while monitoring $S_{v v}$. Figure 2(a) shows this measurement at room temperature for microscope powers $P_{m}=1,2$, and $3 \mathrm{~mW}$. The mechanical frequency $f_{m}\left(z_{f}\right)=f_{m}^{o}+\Delta f_{m}\left(z_{f}\right)$ decreases as the microscope is focused on the nanobeam, consistent with optical heating of a compressively stressed device [45]. $\Delta f_{m}$ follows a profile reminiscent of the $z_{f}$ dependence of the microscope laser intensity, providing a measure of the $I_{f}\left(z_{f}\right)$ profile that can be input to the model in Eq. (1), as discussed below. The asymmetry and oscillations in $\Delta f_{m}\left(z_{f}\right)$ are related to aberrations from the cryostat window [51]. In general, $\Delta f_{m}$ is also affected by dynamic photothermal and dynamic and static optical-gradient-force effects. However, they are predicted to be smaller than the observed $\left|\Delta f_{m}\right|[45]$.

The influence of the microscope on the nanobeam dynamics is revealed dramatically in Fig. 2(a) near $z_{f}=$ $15 \mu \mathrm{m}$, where for $P_{m}=2$ and $3 \mathrm{~mW}$, the peak value of $S_{v v}$ increases, indicating nanomechanical self-oscillation, and $f_{m}$ shifts due to nonlinear nanomechanical effects related to large-amplitude motion [45]. To analyze this quantitatively, the measured $\Delta \Gamma_{m}\left(z_{f}\right)$ is plotted in Fig. 2(b), showing that motion is either damped $\left(\Delta \Gamma_{m}>0\right)$ or amplified $\left(\Delta \Gamma_{m}<0\right)$ depending on the microscope focus: the sign of $\Delta \Gamma_{m}$ changes as the focus is scanned from above to below the nanobeam. Near $z_{f}=15 \mu \mathrm{m}, \Gamma_{m} \sim 0$, the nanobeam enters a regime of self-oscillation, in agreement with the increase in the $S_{v v}$ peak amplitude.

This behavior illustrates a key feature of this system: the dependence of the sign of $\Delta \Gamma_{m}\left(z_{f}\right)$ on the microscope intensity gradient. By fitting the data in Fig. 2(b) with the model from Eq. (1), the relative contribution from the microscope gradient and the etalon are extracted. This requires expanding the intensity gradient,

$$
\frac{d I}{d z}=-\chi\left(\frac{d I_{f}\left(z_{f}\right)}{d z_{f}}-I_{f}\left(z_{f}\right) \frac{1}{\chi} \frac{d \chi}{d z_{s}}\right),
$$

and inferring $d I_{f} / d z_{f}$ and $I_{f}\left(z_{f}\right)$ from $\Delta f_{m}\left(z_{f}\right)$ to within a proportionality constant. In addition to this constant, the fit requires a fitting parameter $\propto \chi$ that governs the relative contributions of the intensity gradient and the etalon terms in Eq. (2). The contributions from these two terms are plotted in Fig. 2(b), showing that in our experiment the microscope gradient is the dominant factor while the smaller etalon contribution damps the mechanical motion and shifts the zero of $\Delta \Gamma_{m}\left(z_{f}\right)$. The imperfect fits reveal the approximate nature of the model. For example, it is possible that the microscope position and the etalon response, which in general is a standing wave pattern, are not entirely separable. In future, detailed numerical simulations of the microscope field and its interaction with the nanobeam and the surrounding diamond structure would provide additional insight into optimization of the strength 

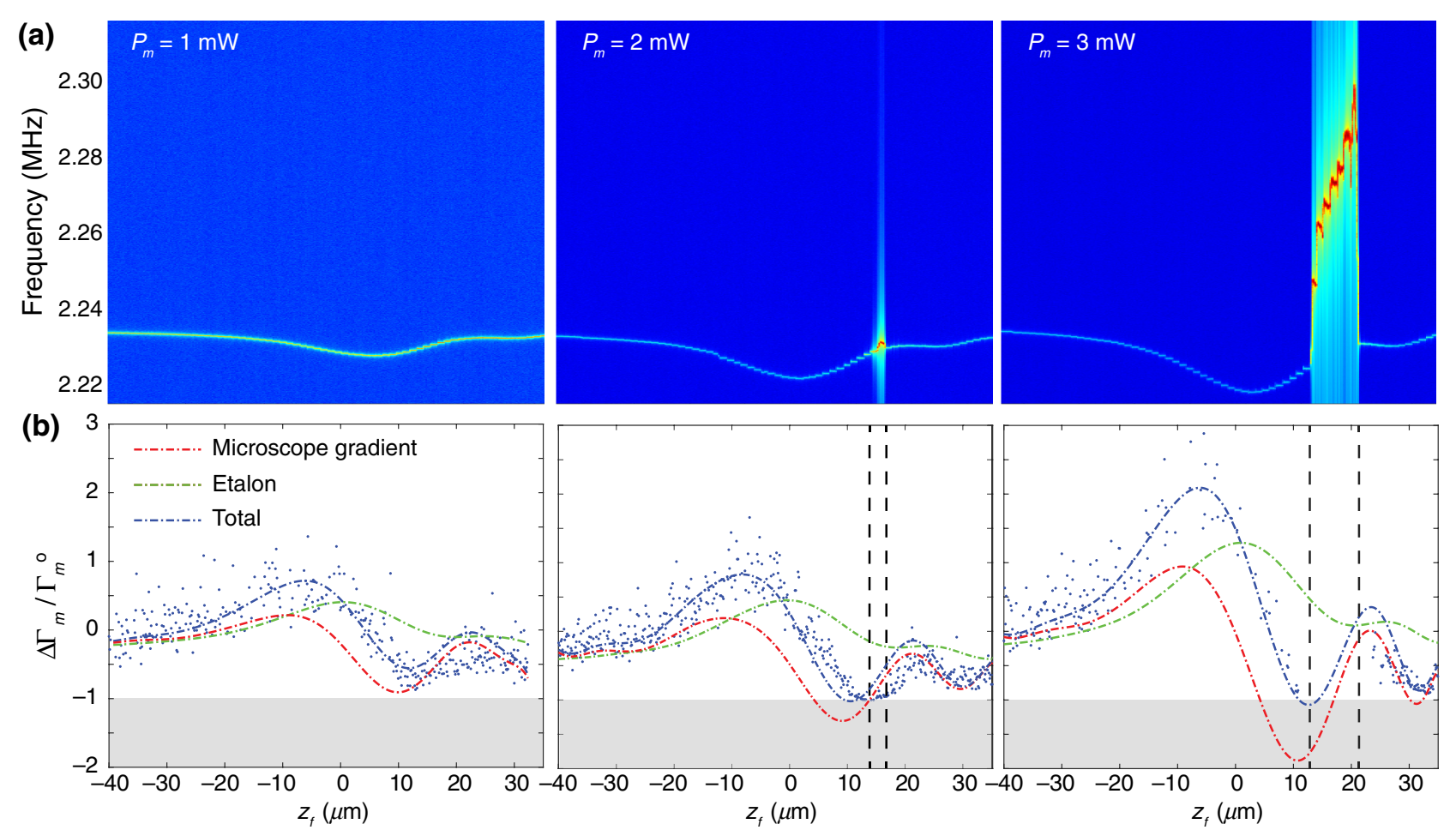

FIG. 2. (a) A spectrograph showing the power spectral density of the $\mathbf{v}_{1}$ nanobeam mode as a function of the microscope focus height, for varying green laser power. The sample is at room temperature. A negative $z_{f}$ indicates that the focal position is below the nanobeam. (b) Optomechanical damping of $\mathbf{v}_{1}$, normalized by its intrinsic dissipation rate, as a function of the focal height and varying power. The fits are from the model in Eq. (1), input with a normalized $I\left(z_{f}\right)$ profile derived from $\Delta f_{m}$. Contributions from the etalon and microscope gradient terms in Eq. (2) are shown. The shaded regions indicate where optomechanical damping will cause self-oscillation.

of the gradient contribution and minimization of the etalon contribution.

The amplification and damping is further analyzed in Fig. 3, which plots the rms amplitude for varying $z_{f}$ and $P_{m}$, extracted from the area under $S_{v v}$ normalized by the thermomechanical-vibration amplitude in the absence of the microscope field [52]. When $P_{m}=3 \mathrm{~mW}$, the selfoscillations reach close to $100 \mathrm{~nm}, 3$ orders of magnitude greater than the intrinsic thermal motion of the nanobeam. In contrast, when the microscope position is set to $z_{f} \sim$ $-5 \mu \mathrm{m}$, the thermal motion of the nanobeam is damped, cooling the resonance to $T_{\text {eff }} \sim 80 \mathrm{~K}$ from the sample temperature $T_{s}=300 \mathrm{~K}$, as shown in the inset to Fig. 3 . This inference of the temperature from the resonance area is found to be consistent with $T_{\text {eff }}=T_{s} /\left(1+\Delta \Gamma_{m} / \Gamma_{m}^{o}\right)$ predicted from $\Delta \Gamma_{m}$ in Fig. 2(b) [20].

\section{CRYOGENIC OPERATION}

\section{A. Low-power self-oscillations and cooling}

The impact of back action is increased in cryogenic conditions, where the intrinsic mechanical dissipation of the diamond resonator is reduced. The improvement in device performance at low temperature is illustrated in Fig. 1(b), which shows that $Q_{m}^{o}$ increases by an order of magnitude when the sample temperature is lowered from $300 \mathrm{~K}$ to $5 \mathrm{~K}$. As a result, for a given optomechanical back action $\left|\Delta \Gamma_{m}\right|$, which is nominally independent of the temperature and $Q_{m}^{o}$, the relative change in mechanical dissipation, $\left|\Delta \Gamma_{m} / \Gamma_{m}^{o}\right| \propto Q_{m}^{o}$, increases. This lowers the power required for optomechanical self-oscillation $\left(\Delta \Gamma_{m} / \Gamma_{m}^{o}=\right.$ $-1)$ or cooling to a desired $T_{\text {eff. }}$.

Figures 4(a) and 4(b) illustrate this effect by showing $S_{v v}$ and the mechanical-vibration amplitude, respectively, at $T_{s}=5 \mathrm{~K}$, for varying $z_{f}$. In these measurements, the microscope power is reduced to $P_{m}=150 \mu \mathrm{W}$ and $60 \mu \mathrm{W}$, respectively. Despite the order-of-magnitude lower $P_{m}$ compared to the room-temperature measurements in Figs. 2 and 3, self-oscillations with comparable amplitudes are observed.

A tantalizing prospect, given the increase in $Q_{m}^{o}$ in the nanobeam at cryogenic temperature, is optomechanical cooling: for the $\mathbf{v}_{1}$ mode, $T_{\text {eff }} \approx 0.3 \mathrm{~K}$ is naively expected at $T_{s}=5 \mathrm{~K}$ from the $\Delta \Gamma_{m}$ observed at room temperature in Fig. 2(b). Figure 4(c) compares $S_{v v}$ of this mode at $T_{\text {eff }}=$ $5 \mathrm{~K}$ with and without the $3-\mathrm{mW}$ microscope field turned on. With the field on and the focus optimized to maximize damping, $Q_{m} \sim 3340$ is inferred from the resonance line 


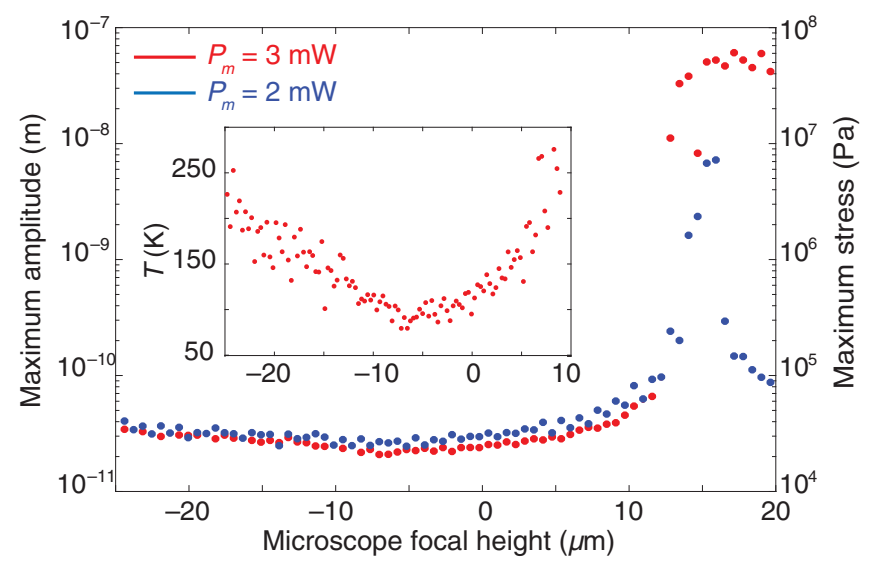

FIG. 3. The amplitude (left axis) and maximum internal dynamic stress (right axis) of the $\mathbf{v}_{1}$ mode at room temperature as a function of the microscope focal plane height for $2-\mathrm{mW}$ and $3-\mathrm{mW}$ microscope power. The sample is at room temperature. When the nanobeam self-oscillates, a maximum stress just below approximately $100 \mathrm{MPa}$ near the nanobeam clamping points can be realized. The inset shows the effective normal-mode temperature for 3-mW microscope power as a function of the microscope height, scanning through the regime of maximum damping.

width. Although this is a 2-orders-of-magnitude increase in line width, the corresponding measured area under $S_{v v}$ is only reduced by a factor of 2.4 by the microscope field, resulting in $T_{\text {eff }}=2.2 \mathrm{~K}$.

The discrepancy between the large broadening of the resonance and the comparatively modest change in $T_{\text {eff }}$ can arise from several sources. At $T_{s}=5 \mathrm{~K}$, the specific heat of diamond is 4 orders of magnitude smaller than at room temperature. As a result, the microscope field can more easily increase the bath temperature of the nanobeam, counteracting cooling via optomechanical damping. Additional line-width broadening could arise from fluctuations of the mechanical resonance frequency induced by the microscope field. Comparing the resonance line width for $P_{m}=3 \mathrm{~mW}$ at $T_{s}=5 \mathrm{~K}$ in Fig. 4(c) with the corresponding maximum room-temperature line width in Fig. 2(b), we can infer that $\left.\Delta \Gamma_{m}\right|_{T_{s}=5 \mathrm{~K}} /\left.\Delta \Gamma_{m}\right|_{T_{s}=300 \mathrm{~K}} \sim 5$ if the damping rate is assumed to be proportional to the line width. Such an enhancement in damping at low temperature requires that photothermal coupling increases in cryogenic conditions; for example, due to changes in the compressive stress of the nanobeam [45]. However, the measurements discussed below reveal that other mechanisms can contribute significantly to line-width broadening at cryogenic temperatures.

\section{B. In-plane mode excitation and line broadening}

The higher $Q_{m}^{o}$ of the device at cryogenic temperatures also enables excitation of in-plane nanobeam motion. This is shown in Fig. 5, which plots $S_{v v}$ of the in-plane fundamental $\mathbf{h}_{1}$ resonance near $f_{m}=3.0 \mathrm{MHz}$ as a function
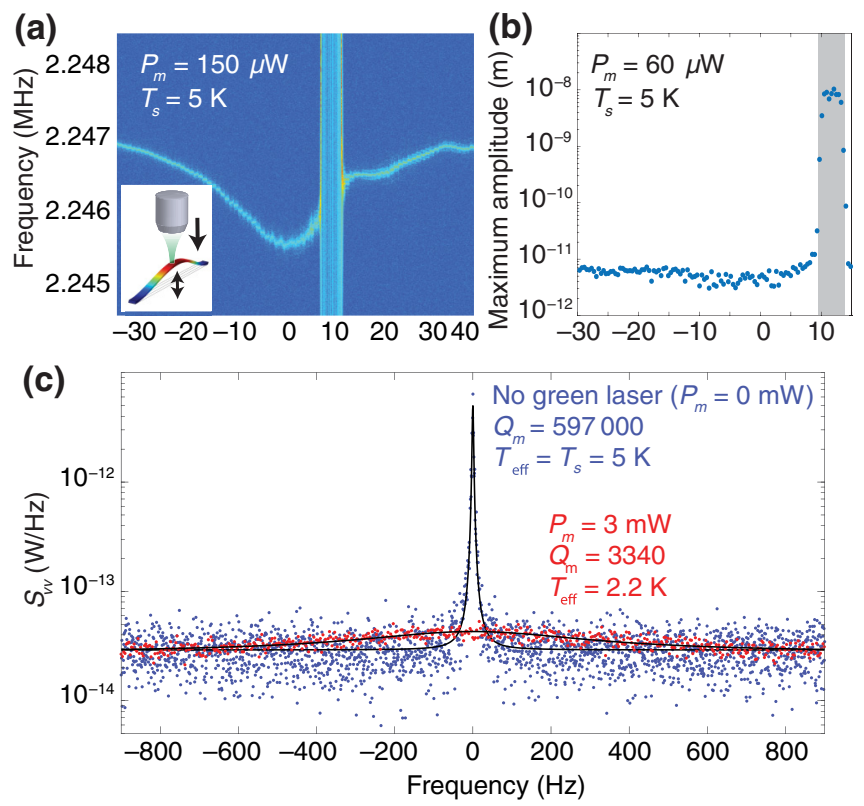

FIG. 4. The nanobeam-microscope optomechanics at cryogenic temperature $T_{s}=5 \mathrm{~K}$ : (a) a spectrograph and (b) the oscillation amplitude of the $\mathbf{v}_{1}$ nanobeam-mode motion for varying focal plane height, detected via fiber-taper transmission. (c) The power spectral density of the $v_{1}$-mode motion detected by fiber-taper transmission with the microscope off $\left(P_{m}=0 \mathrm{~mW}\right)$ and on $\left(P_{m}=3 \mathrm{~mW}\right)$, with $z_{f}$ optimized to maximize $\Delta \Gamma_{m}$.

of the lateral $(x)$ displacement of the objective for $P_{m}=$ $450 \mu \mathrm{W}$. The $4 \mu \mathrm{m} x$ scan length is smaller than the $z$ scans owing to the tight lateral focus of the microscope in comparison to its depth of focus. Self-oscillation occurs near $x=0.75 \mu \mathrm{m}$, while for negative $x$ damping is observed. This asymmetric optomechanical response indicates that the nanobeam deflects laterally in a fixed direction when heated, independent of whether the focus is on the right or left side of the nanobeam.

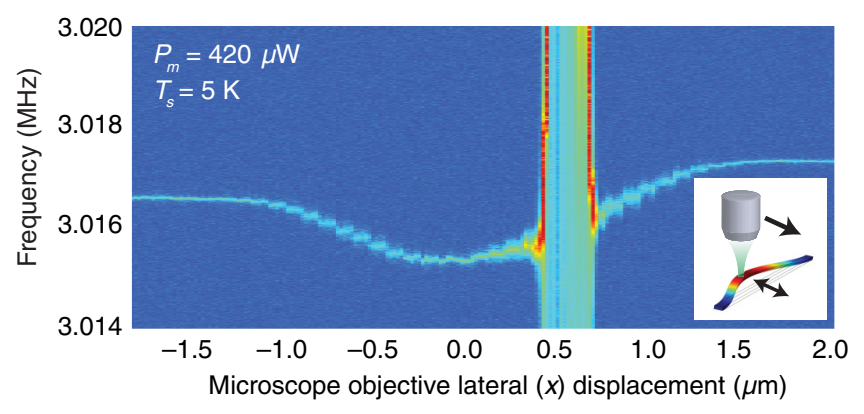

FIG. 5. Exciting in-plane motion: a spectrograph of the fundamental in-plane $\mathbf{h}_{1}$ nanobeam-mode motion for a varying lateral focal spot position, detected via fiber-taper transmission. $P_{m}=$ $420 \mu \mathrm{W}$ and $T_{s}=5 \mathrm{~K}$. 

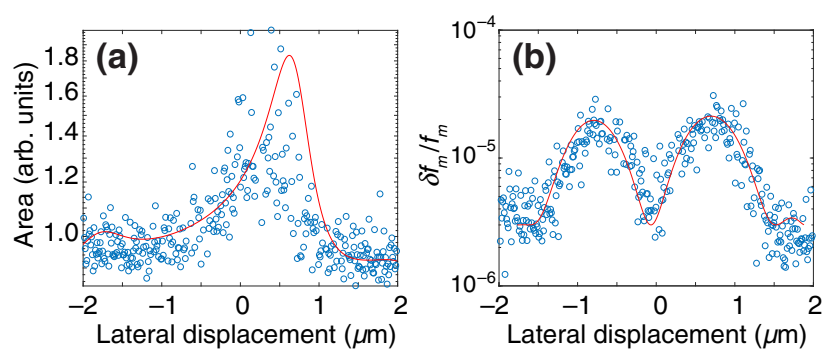

FIG. 6. Competition between optomechanical back action and thermomechanical line-width broadening. (a) The area under the $\mathbf{h}_{1}$ nanobeam-mode spectrum for a varying lateral focal spot position. (b) The corresponding line width extracted by fits to the mode spectrum. Measurements at $P_{m}=200 \mu \mathrm{W}$ and $T_{s}=5 \mathrm{~K}$.

Further analysis of the dependence of the $\mathbf{h}_{1}$ resonance dynamics on the microscope field confirm that mechanisms in addition to optomechanical back action broaden the mechanical line shape. Figure 6(a) plots the resonance area $\left(\propto T_{\text {eff }}\right)$ for varying microscope displacement along $x$ with $P_{m} \sim 200 \mu \mathrm{W}$ set below the threshold for selfoscillation. This clearly illustrates the asymmetric response of the optomechanical damping as a function of $x$ and is consistent with the self-oscillation data in Fig. 5. In contrast, the measured line width, plotted in Fig. 6(b), varies symmetrically with $x$. Broadening is maximized when the nanobeam is positioned adjacent to the microscope focus, where the lateral gradient of the microscope field intensity is strong. This broadening occurs even when the nanobeam motion is being amplified. These observations suggest that displacements of the nanobeam relative to the microscope focus are causing spectral diffusion of $f_{m}$.

To better understand the dependence of the mode area $(A)$ and the measured line width $(\delta f)$ on the microscope position, we fit the $x$-dependent data in Fig. 6 with

$$
\begin{aligned}
& A(x)=A_{0}\left[1+\alpha_{1} \frac{I(x)}{1+\beta \frac{d I}{d x}}\right], \\
& \delta f(x)=\delta f_{o}\left[1+\alpha_{2}\left|\frac{d I}{d x}\right|\right],
\end{aligned}
$$

where the fitting parameter $\alpha_{1}$ describes the increase in $T_{s}$ from the microscope field and parameter $\beta$ describes the strength of the photothermal optomechanical back action and its effect on $\Delta \Gamma$. The fitting parameter $\alpha_{2}$ describes a contribution to spectral broadening resulting from the overlap of the nanobeam with the gradient of the microscope field intensity; for example, that manifest due to variations in the position of the focal spot relative to the nanobeam. The fits in Fig. 6 are created by assuming that $I(x)$ is directly proportional to the measured $\Delta f_{m}(x)$, similar to the room-temperature analysis in Fig. 2(b).
This model is in good agreement with the data and confirms that in addition to optomechanical damping, the line width is being broadened by additional mechanisms described here phenomenologically by nonzero $\alpha_{2}$. Further investigation into the source of this broadening is required. For example, measurements of the Allan variance of the mechanical frequency will reveal the time scale over which it is fluctuating and provide an insight into the nature of any technical noise affecting it. Time-domain measurements of mechanical ring-down will provide a direct measurement of $\Gamma_{m}$. Together with power-dependent measurements of $\delta f_{m}$, this may allow improvement of the efficiency of the photothermal cooling process in cryogenic conditions.

Note that $T_{\text {eff }}$ inferred from the area of $S_{v v}$ is unaffected by spectral diffusion [53] and that at room temperature we observe close agreement between $T_{\text {eff }}$ extracted from $A$ and $\delta f_{m}$, respectively. This indicates that the spectral broadening by the microscope is specific to the cryogenic measurements reported here.

\section{DISCUSSION}

The potential of this system for spin optomechanics is significant. Confocal microscopes are used for diamond spin spectroscopy, making this approach suited for controlling the coupling of phonons and spins. For the self-oscillations reported here, dynamic stress fields of approximately $100 \mathrm{MPa}$ are predicted (COMSOL), as shown in Fig. 3. This corresponds to a spin-stress coupling rate $G_{g} / 2 \pi \sim 1 \mathrm{MHz}$ and $G_{e} / 2 \pi \sim 100 \mathrm{THz}$, for the ground and excited states, respectively, of a negatively charged $\mathrm{N}-V$ center, which are comparable to coupling rates in piezo-based stress-manipulation experiments [22]. Optomechanical spin control will provide a path toward creating a quantum transducer $[30,34]$ for coupling photons to spins without direct optical-color-center transitions, enabling interfacing telecommunication photons with spin quantum memories [54].

\section{CONCLUSION}

In conclusion, we demonstrate optomechanical control of a diamond resonator using a microscope, observing tunable optomechanical damping and amplification of a nanomechanical resonator without a cavity, etalon, or other external feedback component. Using this tunable optomechanical damping, we cool the fundamental mode of the nanobeam to below $80 \mathrm{~K}$ and amplify its motion sufficiently for spin-phonon coupling at rates that exceed relevant spin decoherence rates [22,54]. We also study the interplay between the nanomechanical resonator of the microscope field at low temperature, providing a jumpingoff point for future studies of photothermal cooling in cryogenic environments. 


\section{ACKNOWLEDGMENTS}

We thank Aaron Hryciw, J. P. Hadden, and M. Mitchell for assistance. This work was supported by the Natural Sciences and Engineering Research Council (NSERC) (Discovery and Research Tools and Instruments), the CFI, AITF, and the NRC.

[1] A. Ashkin, Acceleration and Trapping of Particles by Radiation Pressure, Phys. Rev. Lett. 24, 156 (1970).

[2] L. Scientific, B. Abbott, R. Abbott, T. Abbott, F. Acernese, K. Ackley, C. Adams, T. Adams, P. Addesso, and R. Adhikari et al., Gw170104: Observation of a 50-SolarMass Binary Black Hole Coalescence at Redshift 0.2, Phys. Rev. Lett. 118, 221101 (2017).

[3] S. Gröblacher, K. Hammerer, M. R. Vanner, and M. Aspelmeyer, Observation of strong coupling between a micromechanical resonator and an optical cavity field, Nature 460, 724 (2009).

[4] J. Chan, T. P. M. Alegre, A. H. Safavi-Naeini, J. T. Hill, A. Krause, S. Groblacher, M. Aspelmeyer, and O. Painter, Laser cooling of a nanomechanical oscillator into its quantum ground state, Nature 478, 89 (2011).

[5] J. D. Cohen, S. M. Meenehan, G. S. MacCabe, S. Gröblacher, A. H. Safavi-Naeini, F. Marsili, M. D. Shaw, and O. Painter, Phonon counting and intensity interferometry of a nanomechanical resonator, Nature 520, 522 (2015).

[6] R. Riedinger, S. Hong, R. A. Norte, J. A. Slater, J. Shang, A. G. Krause, V. Anant, M. Aspelmeyer, and S. Gröblacher, Non-classical correlations between single photons and phonons from a mechanical oscillator, Nature 530, 313 (2016).

[7] V. Sudhir, R. Schilling, S. A. Fedorov, H. Schütz, D. J. Wilson, and T. J. Kippenberg, Quantum Correlations of Light from a Room-Temperature Mechanical Oscillator, Phys. Rev. X 7, 031055 (2017).

[8] T. P. Purdy, K. E. Grutter, K. Srinivasan, and J. M. Taylor, Quantum correlations from a room-temperature optomechanical cavity, Science 356, 1265 (2017).

[9] R. Riedinger, A. Wallucks, I. Marinković, C. Löschnauer, M. Aspelmeyer, S. Hong, and S. Gröblacher, Remote quantum entanglement between two micromechanical oscillators, Nature 556, 473 (2018).

[10] G. Anetsberger, E. Gavartin, O. Arcizet, Q. Unterreithmeier, E. Weig, M. Gorodetsky, J. Kotthaus, and T. Kippenberg, Measuring nanomechanical motion with an imprecision below standard quantum limit, Phys. Rev. A 82, 061804 (2010).

[11] E. Gavartin, P. Verlot, and T. Kippenberg, A hybrid onchip optomechanical transducer for ultrasensitive force measurements, Nat. Nanotech. 7, 509 (2012).

[12] S. Forstner, S. Prams, J. Knittel, E. van Ooijen, J. Swaim, G. Harris, A. Szorkovszky, W. Bowen, and H. RubinszteinDunlop, Cavity Optomechanical Magnetometer, Phys. Rev. Lett. 108, 120801 (2012).

[13] M. Wu, N. L.-Y. Wu, T. Firdous, F. F. Sani, J. E. Losby, M. R. Freeman, and P. E. Barclay, Nanocavity optomechanical torque magnetometry and radiofrequency susceptometry, Nat. Nanotechnol. 12, 127 (2017).

[14] A. H. Safavi-Naeini, T. M. Alegre, J. Chan, M. Eichenfield, M. Winger, Q. Lin, J. T. Hill, D. Chang, and O. Painter, Electromagnetically induced transparency and slow light with optomechanics, Nature 472, 69 (2011).

[15] S. Weis, R. Rivière, S. Deléglise, E. Gavartin, O. Arcizet, A. Schliesser, and T. J. Kippenberg, Optomechanically induced transparency, Science 330, 1520 (2010).

[16] C. Dong, V. Fiore, M. C. Kuzyk, and H. Wang, Optomechanical dark mode, Science 338, 1609 (2012).

[17] Y. Liu, M. Davanço, V. Aksyuk, and K. Srinivasan, Electromagnetically Induced Transparency and Wideband Wavelength Conversion in Silicon Nitride Microdisk Optomechanical Resonators, Phys. Rev. Lett. 110, 223603 (2013).

[18] D. P. Lake, M. Mitchell, Y. Kamaliddin, and P. E. Barclay, Optomechanically induced transparency and cooling in thermally stable diamond microcavities, ACS Photonics 5, 782 (2018).

[19] T. Kippenberg and K. Vahala, Cavity optomechanics: Backaction at the mesoscale, Science 321, 1172 (2008).

[20] M. Aspelmeyer, T. J. Kippenberg, and F. Marquardt, Cavity optomechanics, Rev. Mod. Phys. 86, 1391 (2014).

[21] I. Aharonovich, A. D. Greentree, and S. Prawer, Diamond photonics, Nat. Photon. 5, 397 (2011).

[22] D. Lee, K. W. Lee, J. V. Cady, P. Ovartchaiyapong, and A. C. B. Jayich, Topical review: Spins and mechanics in diamond, J. Opt. 19, 033001 (2017).

[23] E. R. MacQuarrie, T. A. Gosavi, N. R. Jungwirth, S. A. Bhave, and G. D. Fuchs, Mechanical Spin Control of Nitrogen-Vacancy Centers in Diamond, Phys. Rev. Lett. 111, 227602 (2013).

[24] P. Ovartchaiyapong, K. W. Lee, B. A. Myers, and A. C. B. Jayich, Dynamic strain-mediated coupling of a single diamond spin to a mechanical resonator, Nat. Commun. 5, 4429 (2014).

[25] J. Teissier, A. Barfuss, P. Appel, E. Neu, and P. Maletinsky, Strain Coupling of a Nitrogen-Vacancy Center Spin to a Diamond Mechanical Oscillator, Phys. Rev. Lett. 113, 020503 (2014).

[26] A. Barfuss, J. Teissier, E. Neu, A. Nunnenkamp, and P. Maletinsky, Strong mechanical driving of a single electron spin, Nature Phys. 11, 820 (2015).

[27] E. R. MacQuarrie, T. A. Gosavi, A. M. Moehle, N. R. Jungwirth, S. A. Bhave, and G. D. Fuchs, Coherent control of a nitrogen-vacancy center spin ensemble with a diamond mechanical resonator, Optica 2, 233 (2015).

[28] S. Meesala, Y.-I. Sohn, H. A. Atikian, S. Kim, M. J. Burek, J. T. Choy, and M. Lončar, Enhanced Strain Coupling of Nitrogen-Vacancy Spins to Nanoscale Diamond Cantilevers, Phys. Rev. Appl. 5, 034010 (2016).

[29] D. A. Golter, T. Oo, M. Amezcua, I. Lekavicius, K. A. Stewart, and H. Wang, Coupling a Surface Acoustic Wave to an Electron Spin in Diamond via a Dark State, Phys. Rev. X 6, 041060 (2016).

[30] D. A. Golter, T. Oo, M. Amezcua, K. A. Stewart, and H. Wang, Optomechanical Quantum Control of a NitrogenVacancy Center in Diamond, Phys. Rev. Lett. 116, 143602 (2016). 
[31] S. Maity, L. Shao, S. Bogdanović, S. Meesala, Y.-I. Sohn, N. Sinclair, B. Pingault, M. Chalupnik, C. Chia, and L. Zheng et al., Coherent acoustic control of a single silicon vacancy spin in diamond, arXiv:1910.09710 (2019).

[32] M.-A. Lemonde, S. Meesala, A. Sipahigil, M. J. A. Schuetz, M. D. Lukin, M. Loncar, and P. Rabl, Phonon Networks with Silicon-Vacancy Centers in Diamond Waveguides, Phys. Rev. Lett. 120, 213603 (2018).

[33] M. C. Kuzyk and H. Wang, Phononic quantum networks of solid-state spins with alternating and frequency-selective waveguides, arXiv:1804.07862 (2018).

[34] M. J. A. Schuetz, E. M. Kessler, G. Giedke, L. M. K. Vandersypen, M. D. Lukin, and J. I. Cirac, Universal Quantum Transducers Based on Surface Acoustic Waves, Phys. Rev. X 5, 031031 (2015).

[35] D. Rugar and P. Grütter, Mechanical Parametric Amplification and Thermomechanical Noise Squeezing, Phys. Rev. Lett. 67, 699 (1991).

[36] R. A. Barton, I. R. Storch, V. P. Adiga, R. Sakakibara, B. R. Cipriany, B. Ilic, S. P. Wang, P. Ong, P. L. McEuen, and J. M. Parpia et al., Photothermal self-oscillation and laser cooling of graphene optomechanical systems, Nano Lett. 12, 4681 (2012).

[37] Z. Yie, K. Turner, N. Miller, and S. Shaw, in Proceedings of the 13th Hilton Head Solid State Sensors and Actuators Conference (2010).

[38] Q. Lin, J. Rosenberg, X. Jiang, K. J. Vahala, and O. Painter, Mechanical Oscillation and Cooling Actuated by the Optical Gradient Force, Phys. Rev. Lett. 103, 103601 (2009).

[39] O. Arcizet, P. F. Cohadon, T. Briant, M. Pinard, and A. Heidmann, Radiation-pressure cooling and optomechanical instability of a micromirror, Nature 444, 71 (2006).

[40] S. Gigan, H. R. Böhm, M. Paternostro, F. Blaser, G. Langer, J. B. Hertzberg, K. C. Schwab, D. Bäuerle, M. Aspelmeyer, and A. Zeilinger, Self-cooling of a micromirror by radiation pressure, Nature 444, 67 (2006), arXiv:quant-ph/0607068.

[41] C. Metzger, I. Favero, A. Ortlieb, and K. Karrai, Optical self cooling of a deformable Fabry-Perot cavity in the classical limit, Phys. Rev. B 78, 035309 (2008).

[42] C. H. Metzger and K. Karrai, Cavity cooling of a microlever, Nature 432, 1002 (2004).
[43] I. Favero, C. Metzger, S. Camerer, D. König, H. Lorenz, J. P. Kotthaus, and K. Karrai, Optical cooling of a micromirror of wavelength size, Appl. Phys. Lett. 90, 104101 (2007).

[44] D. Ramos, E. Gil-Santos, V. Pini, J. M. Llorens, M. Fernandez-Regulez, A. S. Paulo, M. Calleja, and J. Tamayo, Optomechanics with silicon nanowires by harnessing confined electromagnetic modes, Nano Lett. 12, 932 (2012), pMID: 22268657.

[45] B. Khanaliloo, H. Jayakumar, A. C. Hryciw, D. P. Lake, H. Kaviani, and P. E. Barclay, Single-Crystal Diamond Nanobeam Waveguide Optomechanics, Phys. Rev. X 5, 041051 (2015).

[46] M. Poggio, C. Degen, H. Mamin, and D. Rugar, Feedback Cooling of a Cantilever's Fundamental Mode below $5 \mathrm{mK}$, Phys. Rev. Lett. 99, 017201 (2007).

[47] M. J. Burek, D. Ramos, P. Patel, I. W. Frank, and M. Lončar, Nanomechanical resonant structures in single-crystal diamond, Appl. Phys. Lett. 103, 131904 (2013).

[48] C. P. Michael, M. Borselli, T. J. Johnson, C. Chrystala, and O. Painter, An optical fiber-taper probe for wafer-scale microphotonic device characterization, Opt. Express 15, 4745 (2007).

[49] A. Cleland and M. Roukes, Noise processes in nanomechanical resonators, J. Appl. Phys. 92, 2758 (2002).

[50] W. Li, N. Mingo, L. Lindsay, D. A. Broido, D. A. Stewart, and N. A. Katcho, Thermal conductivity of diamond nanowires from first principles, Phys. Rev. B 85, 195436 (2012).

[51] M. J. Nasse and J. C. Woehl, Realistic modeling of the illumination point spread function in confocal scanning optical microscopy, Josa a 27, 295 (2010).

[52] M. Mitchell, B. Khanaliloo, D. P. Lake, T. Masuda, J. P. Hadden, and P. E. Barclay, Single-crystal diamond lowdissipation cavity optomechanics, Optica 3, 963 (2016).

[53] J. Moser, A. Eichler, J. Güttinger, M. I. Dykman, and A. Bachtold, Nanotube mechanical resonators with quality factors of up to 5 million, Nat. Nanotechnol. 9, 1007 (2014).

[54] P. K. Shandilya, D. P. Lake, M. J. Mitchell, D. D. Sukachev, and P. E. Barclay, Optomechanical interface between telecom photons and spin quantum memory, arXiv:2102.04597 [quant-ph] (2021). 